\title{
Proper orthogonal decomposition of the flow in geometries containing a narrow gap
}

Received: 8 April 2008 / Accepted: 9 June 2009 / Published online: 10 September 2009

(C) Springer-Verlag 2009

\begin{abstract}
Geometries containing a narrow gap are characterized by strong quasi-periodical flow oscillations in the narrow gap region. The above mentioned phenomena are of inherently unstable nature and, even if no conclusive theoretical study on the subject has been published, the evidence shown to this point suggests that the oscillations are connected to interactions between eddy structures of turbulent flows on opposite sides of the gap. These coherent structures travel in the direction of homogeneous turbulence, in a fashion that strongly recalls a vortex street. Analogous behaviours have been observed for arrays of arbitrarily shaped channels, within certain range of the geometric parameters. A modelling for these phenomena is at least problematic to achieve since they are turbulence driven. This work aims to address the use of Proper Orthogonal Decomposition (POD) to reduce the Navier-Stokes equations to a set of ordinary differential equations and better understand the dynamics underlying these oscillations. Both experimental and numerical data are used to carry out the POD.
\end{abstract}

Keywords Eccentric channel $\cdot$ POD $\cdot$ Low-dimensional models

Mathematics Subject Classification (2000) 76D99 - 76D05 · 76D06

\section{List of symbols}

$y^{+} \quad$ Normalized wall distance

$x_{i} \quad$ Cartesian coordinates (vector notation)

$x, y, z \quad$ Cartesian coordinates

u Velocity vector

$u_{i} \quad$ Cartesian velocity components

$u \quad$ Cartesian velocity component in direction $x$

$v \quad$ Cartesian velocity component in direction $y$

$w \quad$ Cartesian velocity component in direction $z$

E. Merzari $(\varangle) \cdot$ H. Ninokata

Research Laboratory for Nuclear Reactors, Tokyo Institute of Technology, N1-5, 2-12-1 O-Okayama,

Meguro-ku, Tokyo 152-8550, Japan

E-mail: merzari@nr.titech.ac.jp

A. Mahmood · M. Rohde

PNR-R3, Delft University of Technology, Mekelweg 15, 2629 JB Delft, The Netherlands

M. Rohde

E-mail: m.rohde@tudelft.nl 


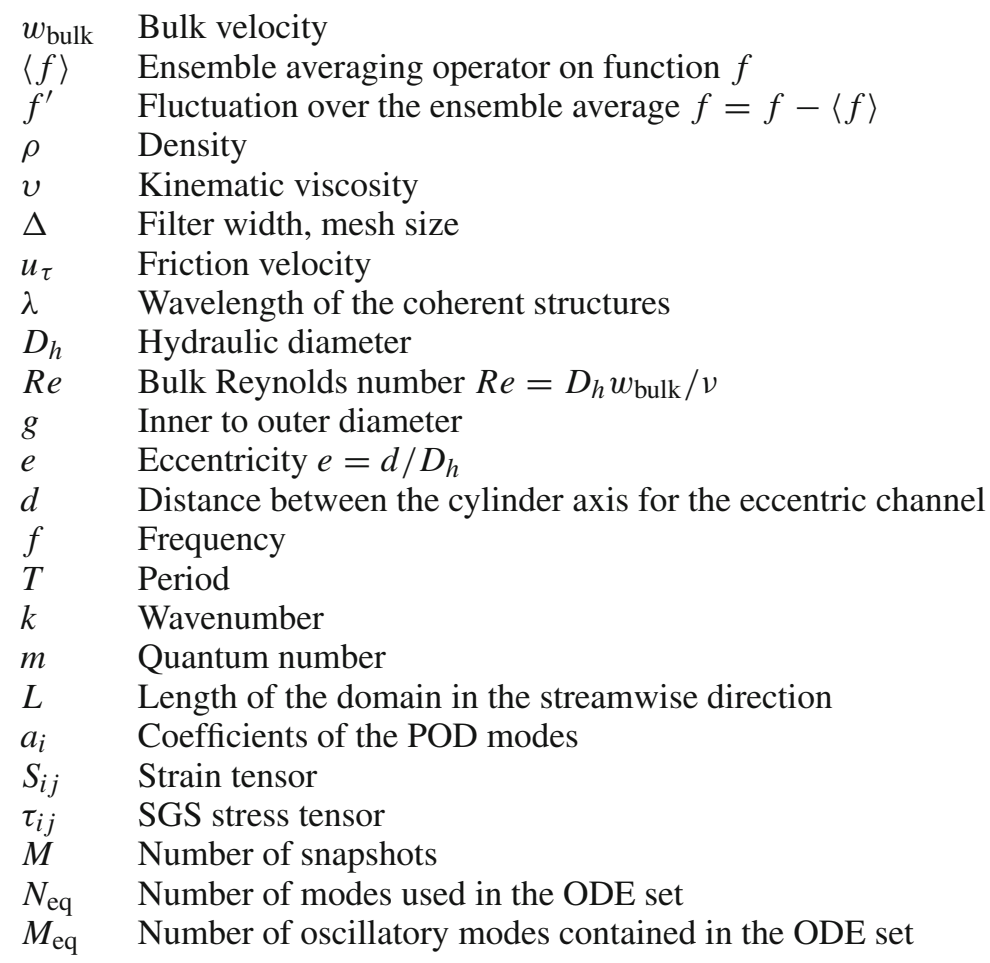

\section{Introduction}

Turbulent flows often exhibit non-obvious behaviours whose prediction remains a challenge. An example is the turbulent flow in closely spaced arrays [1], or, more generally, the turbulent flow in parallel arbitrarily shaped channels connected by a narrow gap [2].

The steady laminar solution of Navier-Stokes equations for these geometries presents a degree of symmetry that is equivalent to the symmetry of the overall system. As an example, let us examine the fully-developed laminar solution for the flow in parallel rectangular subchannels of the same shape connected by a narrow gap (Fig. 1). It is invariant in the axial direction and symmetric to the gap mid-plane (Fig. 1). In the sense of ensemble averaging this fundamental feature is kept by turbulent flows. However, as the analysis does not limit to the averaged statistics and extends to the instantaneous realizations of the flow, the flow is obviously not symmetric.

Moreover, for some shapes of the narrow gap (for a rectangular gap, when the ratio between gap height and gap length is smaller than 2-Fig. 1) large scales structures are observed at the edges of the gap. A flow pulsation is established between the subchannels, whose frequency and spatial distribution are a function of the Reynolds number and the geometry parameters, as they have been reproduced in different experimental facilities. It appears to be a large-scale phenomenon originated by the interacting vortices (or local structures) located in different areas of the domain. We therefore have a symmetric system (the system has reflection symmetry to the gap mid-plane) and a flow that is strongly asymmetric even after integration in time (unless long integration time is taken). Moreover, on the gap mid-plane the Probability Density Function (PDF) of the velocity component parallel to the gap length has a strong non-Gaussian behaviour [3].

At very low Reynolds numbers $(R e<1,000)$ the laminar flow is stable and no oscillations are present. At higher Reynolds numbers coherent structures are observed on the border of the gap. This behaviour might be related to a bifurcation of the laminar steady state that has been observed both computationally and experimentally [4,5]. In fact, experimental results [5] have shown that the onset of the transition to turbulence in geometries containing a narrow gap is characterized by a strong oscillatory behavior of the cross velocity in the gap region [6]. Moreover, the bifurcation can be predicted by linear stability analysis as shown by Merzari et al. [4]. At higher Reynolds numbers, the oscillations begin to be associated to counter-rotating eddies located near the gap-edge [7].

A satisfying modelling, to complement this phenomenological understating, is still lacking. Such a model would be viable in nuclear engineering applications to take into account the enhanced mixing between the channels that these oscillations entail. Basic research is needed to unveil the mechanisms underlying this 

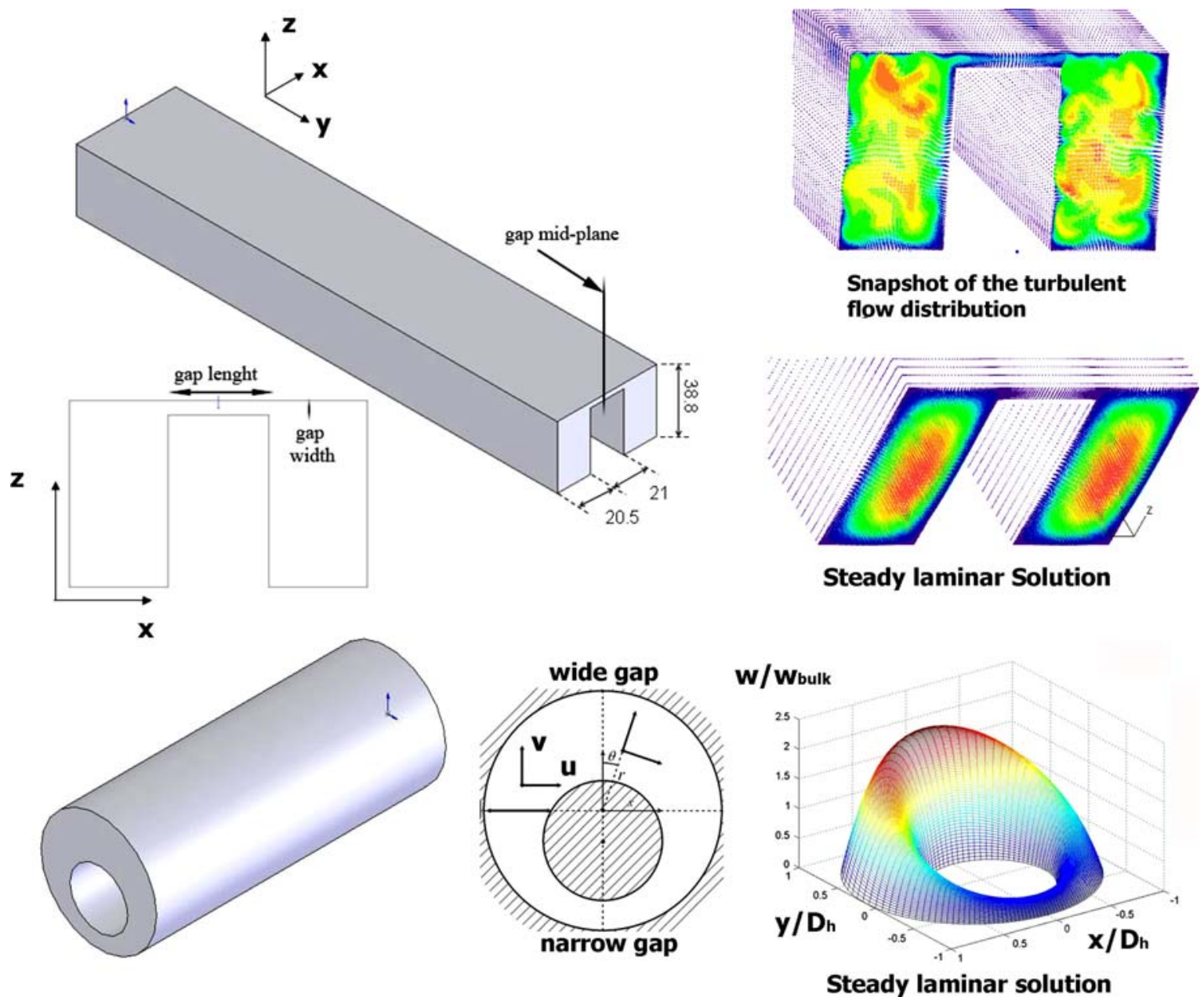

Fig. 1 Geometry and 3D snapshots

phenomenon. Using experimental as well as computational data, this work aims to address this problem through Proper Orthogonal Decomposition (POD) to identify the most significant modes of turbulence and to use Galerkin projection in order to reduce the Navier-Stokes equations to a low-dimensional set of Ordinary Differential Equations (ODEs) [8,9]. By examining the structure of the POD modes and the structure of the coefficient matrices of the low-dimensional model additional insight into the dynamics of the oscillations will be obtained.

\subsection{Proper orthogonal decomposition}

Proper Orthogonal Decomposition has been introduced to the turbulence community by Lumley [10,11]. Lumley proposed to define a coherent structure with functions of the spatial variables that have maximum energy content. That is, coherent structures are (linear combinations of) $\boldsymbol{\sigma}(\mathbf{x})$ which maximize the following expression:

$$
\frac{\left\langle\left(\sigma(\mathbf{x}), \mathbf{u}^{\prime}(\mathbf{x})\right)\right\rangle}{\langle(\boldsymbol{\sigma}(\mathbf{x}), \boldsymbol{\sigma}(\mathbf{x}))\rangle}
$$

In which the expression $(f, g)$ represents the $L^{2}$ product $\int f g d \Omega,\langle\rangle$ the operation of ensemble averaging and $\Omega$ the domain of integration. $\mathbf{u}^{\prime}$ represents the velocity fluctuation toward the average. In fact, it is common to apply POD directly to the velocity fluctuation rather than the complete velocity (thus separating the effect of coherent structures from the mean motion).

If $\sigma(\mathbf{x})$ maximizes (1), it means that if the flow field is projected (in the sense of the $L^{2}$ product) along $\sigma(\mathbf{x})$, the average energy content is larger than if the flow field were to be projected along any other function, 
e.g., a Fourier mode. In the space orthogonal to $\sigma(\mathbf{x})$ the maximization process can be repeated, and in this way a whole set of orthogonal functions $\sigma_{\mathbf{i}}(\mathbf{x})$ can be determined. The power of the POD lies in the fact that the decomposition of the flow field in the POD eigen-functions:

$$
\mathbf{u}^{\prime}(\mathbf{x})=\sum_{i} a_{i} \sigma_{\mathbf{i}}(\mathbf{x})
$$

converges optimally fast in $L^{2}$; i.e., a truncation of $N_{\text {eq }}$ modes in the POD decomposition is the optimal possible truncation for the same number of modes. Each mode is characterized by its energy content, the rank of the modes based on their energy content will be called "quantum number" $m$ in the following [8].

The direct solution of the eigenvalue problem resulting from (1) can be computationally extremely expensive for three-dimensional (3D) turbulence. In fact it would be cumbersome. A possible solution is given by a technique first developed by Sirovich [12]. It is possible to reconstruct the eigenvalue-eigenfunction problem by collecting an adequate number $M$ of realizations of the flow field. This method can be used for ergodic and non-ergodic flows as shown by Aubry et al. [13]. The eigenvalue problem will then be of size $M \times M$ that would be, in general, much more manageable. The value of $M$ for the present work has been determined such as the first 16 eigenvalues for the POD matrix $M \times M$ are the same eigenvalues of the matrix $\sqrt{2} M \times \sqrt{2} M$ (i.e., obtained using $\sqrt{2} M$ snapshots, given by the closest integer to $\sqrt{2} M$ ). Thus, for the chosen number of snapshots $M$, the eigenvalues are considered to be converged.

In this work, two cases will be considered: the flow in an eccentric channel and the flow in two rectangular ducts connected by a narrow gap (Fig. 1). CFD results as well as experimental data have been used to recognize the coherent structures containing the highest quantity of Turbulent Kinetic Energy.

\section{Rectangular channels connected by a narrow gap}

The first case considered is the parallel flow in a U-shaped channel. The layout consists of two rectangular channels connected by a narrow gap (Fig. 1). The flow is in the $x$-direction. The working fluid is water and the bulk Reynolds numbers surveyed by the experiment cover a range from 1,000 to 10,000. The area monitored by Particle Image Velocimetry (PIV) is a $50 \mathrm{~mm} \times 50 \mathrm{~mm}$ plane positioned symmetrically in the middle of the gap. The experimental setup is shown in Fig. 2.

\subsection{Redefinition of the eigenvalue problem for the experimental data}

Since the area monitored is only two-dimensional (2D), POD needs to be applied carefully. Even though POD is a pattern recognition technique, and therefore applicable to every set of data, turbulence is inherently 3D and the results of a 3D POD might have little to do with a 2D POD. However, if turbulence is locally strongly anisotropic (e.g., if turbulence has a two-component behaviour) and the coherent structures are local (i.e., they are contained within the measurement area), it is reasonable to assume that even a 2D POD would be able to yield results in qualitative agreement with a complete 3D POD. To do so the operator of (1) is redefined as follows:

$$
\frac{\left\langle\left(\boldsymbol{\sigma}(\mathbf{x}), \mathbf{u}_{\mathbf{p}}^{\prime}(\mathbf{x})\right)_{p}\right\rangle}{\left\langle(\boldsymbol{\sigma}(\mathbf{x}), \boldsymbol{\sigma}(\mathbf{x}))_{p}\right\rangle}
$$

where $\mathbf{u}_{\mathbf{p}}^{\prime}(\mathbf{x})$ is the velocity vector projected in the measurement plane (therefore, $\left.2 \mathrm{D}\right)$ and $(f, g)_{p}=\int f g d S_{p}$ is the $L^{2}$ product on the measurement plane $S_{p}$. The main hypothesis is that $\mathbf{u}_{\mathbf{p}}^{\prime}(\mathbf{x}) \cdot \mathbf{u}_{\mathbf{p}}^{\prime}(\mathbf{x}) \cong \mathbf{u}^{\prime}(\mathbf{x}) \cdot \mathbf{u}^{\prime}(\mathbf{x}), \forall \mathbf{x} \in$ $S_{p}$, i.e., that the turbulent kinetic energy is distributed mainly in the directions lying in the measurement plane.

Interestingly, a recent numerical work suggests that, in the presence of oscillations, turbulence presents strong anisotropy and a 2D behaviour in the narrow gap [14], thus supporting the validity of the hypothesis made.

The major drawback for this formulation of the POD problem is that the POD eigenfunctions will not be in general divergence free. Therefore, a model based on these equations will not in general respect the continuity equation. 

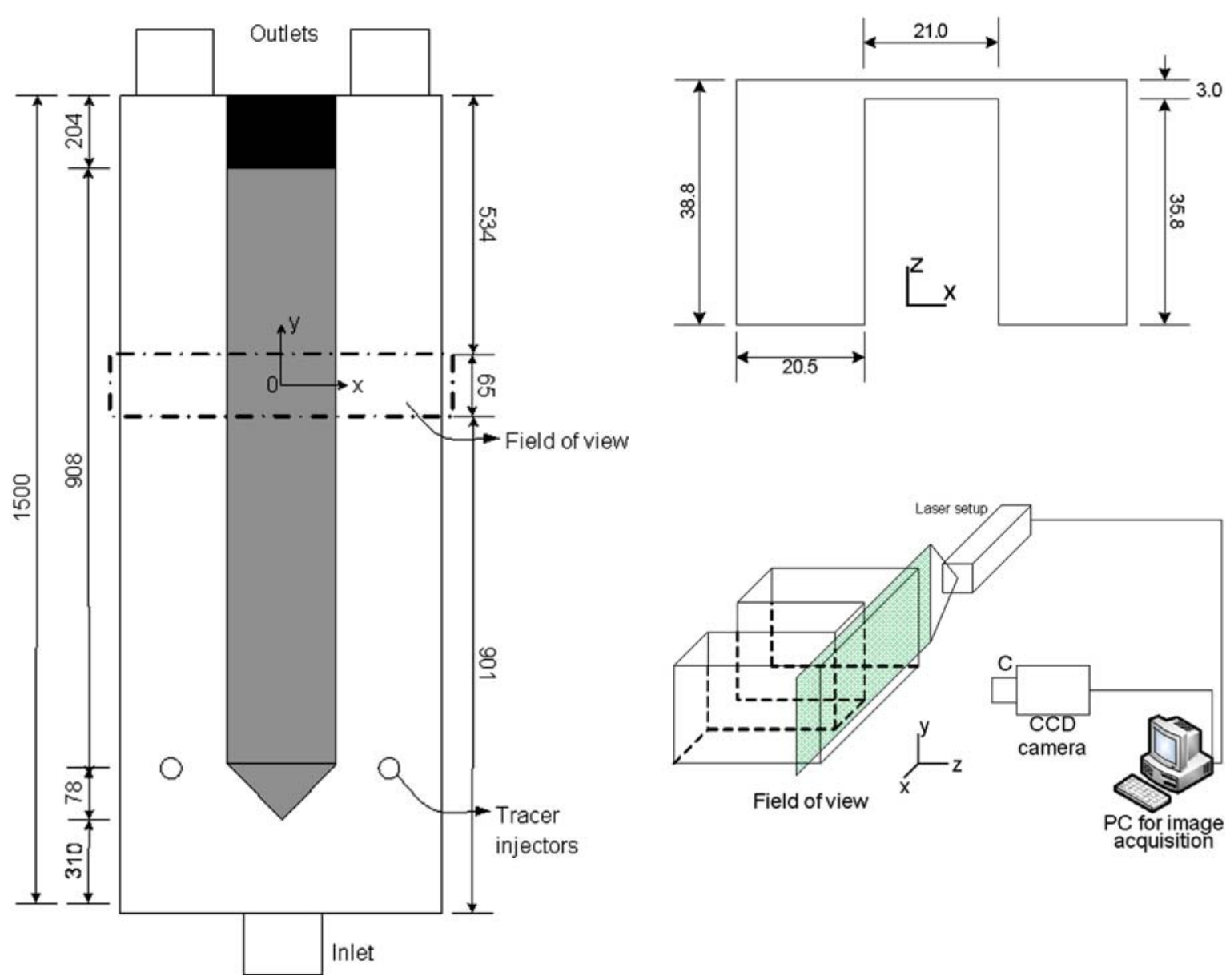

Fig. 2 Experimental setup

\subsection{Computational practices for the CFD calculation}

A Large Eddy Simulation (LES) computation has been carried out for this case at $R e=2,700$. The application of POD on LES data instead of Direct Numerical Simulation (DNS) data does not represent a drawback of the present work, since the modelled scales energy content is inherently incoherent. Moreover, the focus of the present work is on the large scales. In fact, in LES, the small scales of motion, corresponding to the higher part of the Kolgomorov spectrum, are considered to have a nearly universal behaviour and can therefore be modelled. In order to do so a filter operator of the type:

$$
\bar{f}(x)=\int_{-\infty}^{+\infty} G\left(x^{\prime}, x\right) f\left(x^{\prime}\right) \mathrm{d} x^{\prime}
$$

is applied to the Navier-Stokes equations, obtaining the filtered Navier-Stokes equations. The operation introduces six new unknowns $\tau_{i j}$ :

$$
\begin{gathered}
\frac{\partial \bar{u}_{j}}{\partial t}+\frac{\partial \bar{u}_{i} \bar{u}_{j}}{\partial x_{i}}=-\frac{\partial}{\partial x_{i}}\left(\frac{\bar{p}}{\rho} \delta_{i j}+\tau_{i j}\right)+v \frac{\partial^{2} \bar{u}_{i}}{\partial^{2} x_{i}} \\
\frac{\partial \bar{u}_{i}}{\partial x_{i}}=0
\end{gathered}
$$

where the six new unknowns are defined by:

$$
\tau_{i j}=\overline{u_{i} u_{j}}-\bar{u}_{i} \bar{u}_{j}
$$


(a)

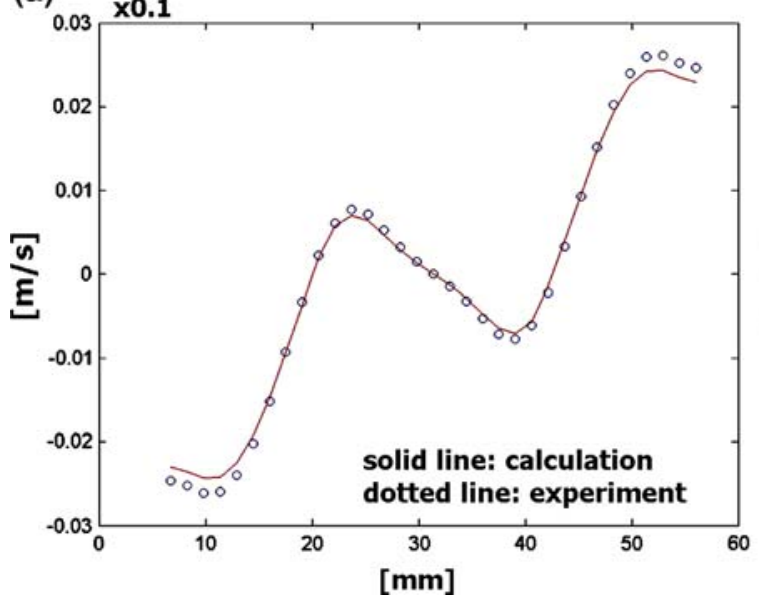

(b)

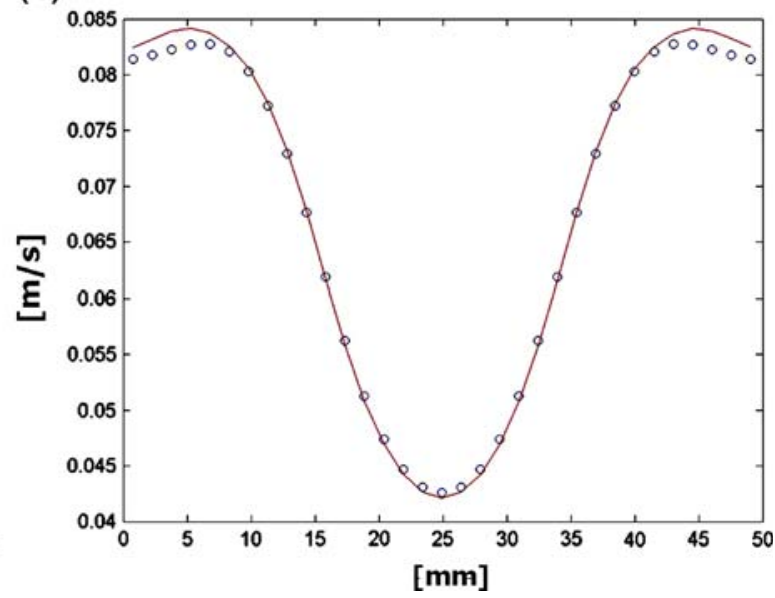

Fig. 3 Comparison between experiment (dots) and calculation (solid line) for the U-shaped channel $(R e=2,700)$. a Averaged cross velocity, b Streamwise velocity. The experimental data in the center of the channels is slightly different from the results of Lexmond et al. [7]. In fact the data shown here has been collected by the present authors on the same experimental facility

these unknowns are dimensionally equivalent to stresses, and they are often modelled within the eddy-viscosity paradigm. In the recent years new approaches have flourished. In the present work, the Smagorinsky model has been used to model the deviatoric part of the stresses, using the following expression:

$$
\tau_{i j}-\tau_{i j} \delta_{i j} \frac{1}{3}=-2\left(C_{s} \Delta\right)^{2}\|S\| S_{i j}
$$

where

$$
\|S\|=\sqrt{2 S_{i j} S_{i j}}
$$

and $\Delta$ is the filter width. Since in practical simulation, no actual filtering is usually performed, its definition is somewhat arbitrary. Several different formulations have been tested and the following definition has been used:

$$
\Delta=\min \left(\Delta_{x}, \Delta_{y}, \Delta_{z}\right)\left(1-\mathrm{e}^{-y^{+} / 25}\right)
$$

which appears to take better into account the effects connected with the adoption of an anisotropic grid and the wall effect. As for the coefficient $C_{s}$ it has been chosen to be constant for the present calculation and equal to 0.1 [15]. Streamwise periodic boundary conditions have been implemented and the length of the computational domain in the streamwise direction has been chosen as equal to $120 \mathrm{~mm}$. The time step for the calculation has been set to $\Delta t=0.00015 \mathrm{~s}$ (with a Courant number lower than 0.1 ) for about 800,000 meshes. The convective fluxes have been discretized through a second-order central scheme while time advancement has been carried out through a Crank-Nicholson scheme. An extensive account of the numerical and modelling practices is given in Merzari et al. [3], along with a detailed discussion of the computational results. Here, only a brief comparison with the experiment will be shown along with the POD.

Figure 3 shows a comparison between experiment and computation for $R e=2,700$. In particular Fig. 3a shows the average cross velocity in the experimental section. It appears that the computational methodology is able to reproduce correctly the secondary flows. Figure $3 \mathrm{~b}$ shows instead the computed streamwise velocity compared with the experimental results. The accuracy in the middle of the gap is excellent. As for the higher moments the peak of rms streamwise velocity is located in the edge of the gap [3], and it has been evaluated in the calculation at $0.263 \mathrm{~m} / \mathrm{s}$ (a value very similar to the one measured experimentally at $R e=2,700$ ).

\subsection{POD of experimental data}

In this section, we will present the results of the problem (3) for the geometry presented at $R e=3,000$ and $R e=2,000$ based on experimental data. A total of 1,600 snapshots have been collected at time intervals of 


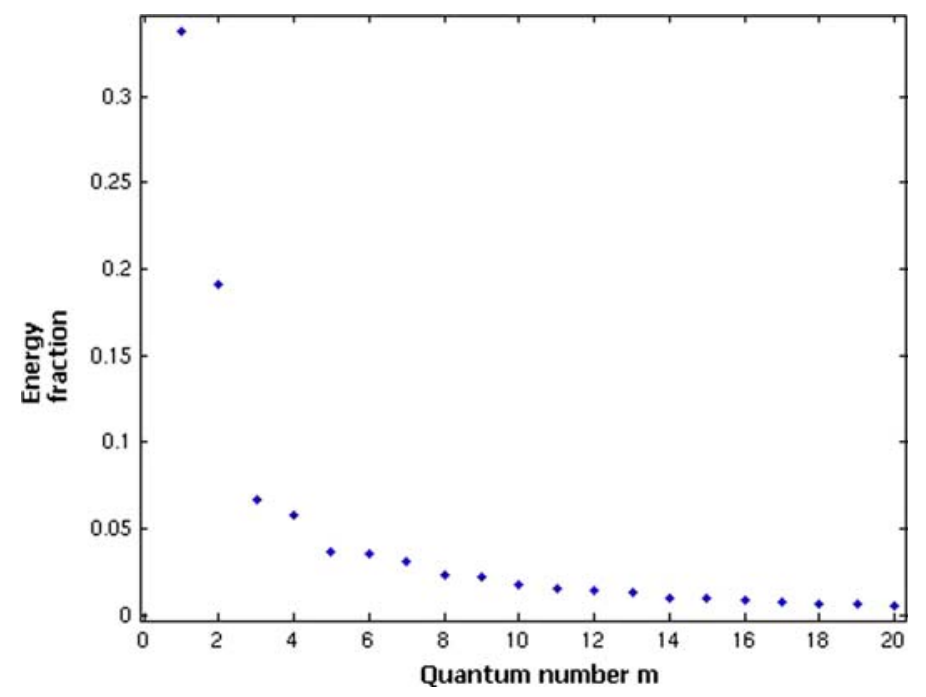

Fig. 4 Energy fraction contained by each POD mode as a function of their quantum number, experimental data for the U-shaped channel $(\operatorname{Re}=2,000)$

(a)

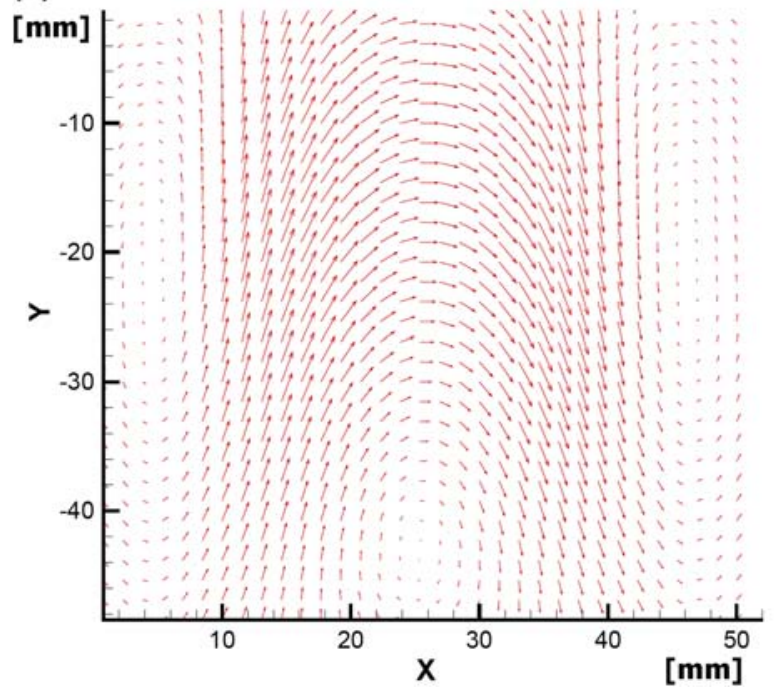

(b)

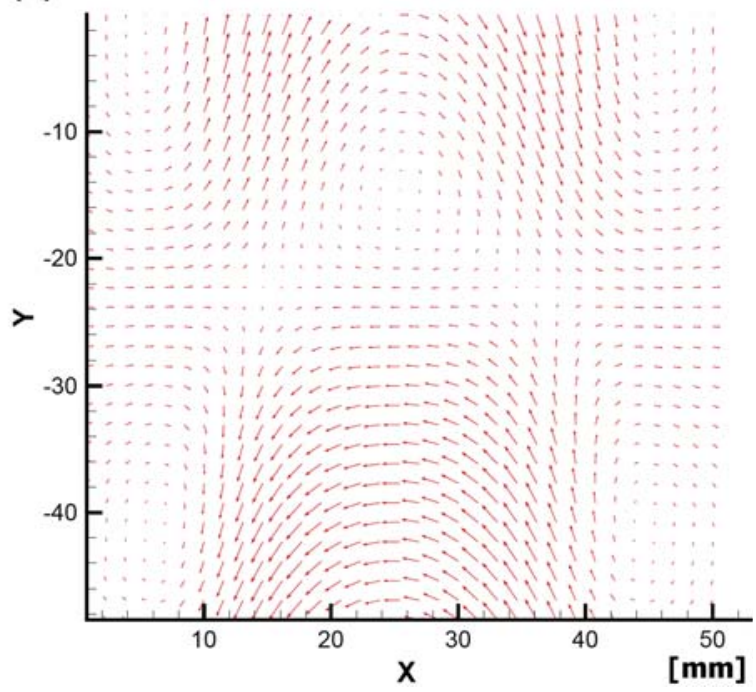

Fig. 5 Most energetic modes, $m=1$ (a) and $m=2(\mathbf{b}), R e=2,000$, experimental data for the U-shaped channel

$0.01 \mathrm{~s}$ and a 3,200 $\times 3,200$ eigenvalue has been assembled and solved (using symmetry considerations). The results are reported in Fig. 4, it is possible to notice that most of the energy is contained in the first eigenmodes (the first 6 eigenmodes contain approximately $75 \%$ of the energy).

Figures 5, 6 and 7 show the most energetic modes. The two most energetic modes ( $m=1$ and $m=2$ ) are evidently the same mode shifted in the axial direction. They represent a traveling wave of the type $\mathbf{u}=$ $\mathbf{u}_{0} \sin \left(2 \pi\left[\frac{x}{\lambda}-\frac{t}{T}\right]\right)$, which can in fact be splitted into two contributions:

$$
\mathbf{u}=\mathbf{u}_{0} \sin \left(\frac{2 \pi}{\lambda} x\right) \sin \left(\frac{2 \pi}{T} t+\frac{\pi}{2}\right)-\mathbf{u}_{0} \sin \left(\frac{2 \pi}{\lambda} x+\frac{\pi}{2}\right) \sin \left(\frac{2 \pi}{T} t\right)
$$

where the two terms differ in the axial direction $x$ by a phase shift of $\pi / 2$. The wavelength of the wave can be evaluated at about $7 \mathrm{~cm}$ by simply measuring the length of the region with negative cross velocity in Fig. 5 and multiplying by a factor of two. The value of the wavelength does not depend upon the Reynolds number and it has been found constant between $R e=3,000$ and $R e=2,000$. Similar considerations apply to the modes with $m=3$ and $m=5$ and several other less energetic modes. 
(a)

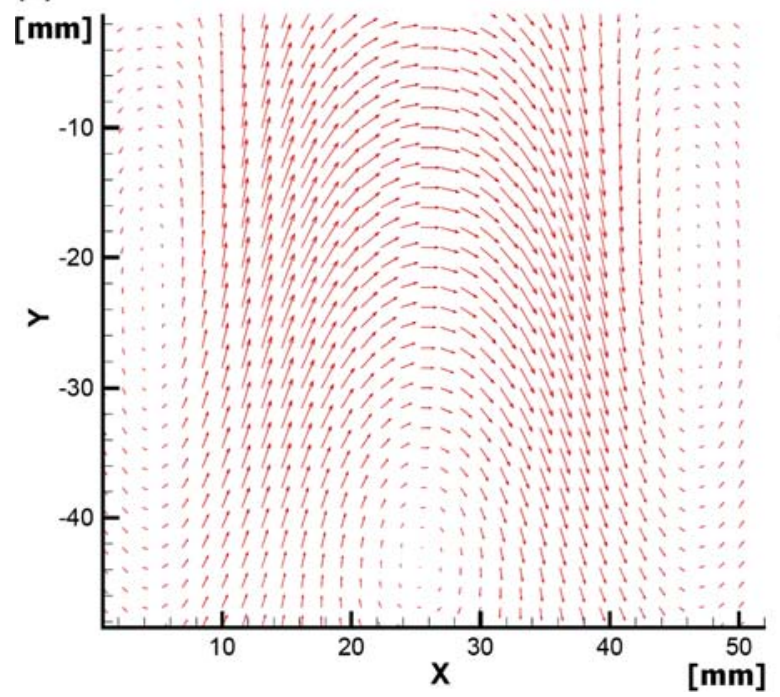

(b)

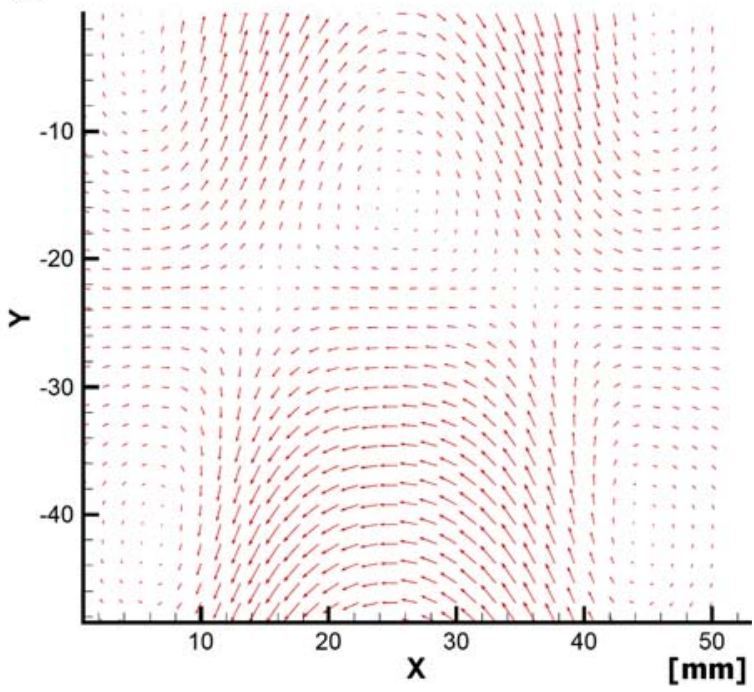

Fig. 6 Modes with quantum number $m=4$ (a) and $m=64$ (b), $R e=2,000$, experimental data for the U-shaped channel

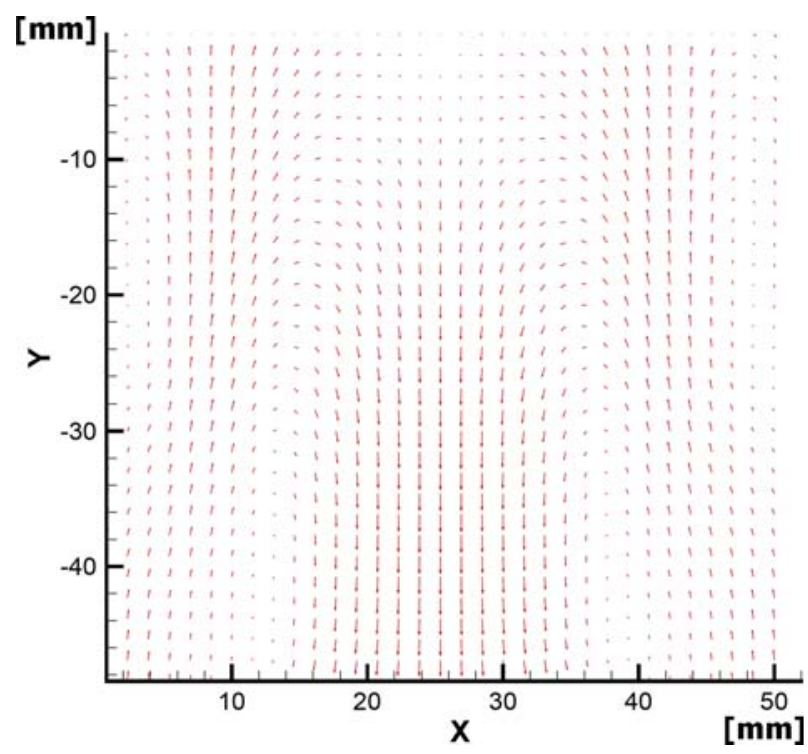

Fig. 7 Mode with $m=5, R e=2,000$, experimental data for the U-shaped channel

The other modes are also responsible for the energy transfer between channels. They do not represent, however, traveling waves. As an example, Fig. 7 shows the mode with $m=4$, containing two vortices on the edge of the gap.

\subsection{POD of CFD data}

For CFD data 1,000 snapshots have been collected at $0.015 \mathrm{~s}$ (100 time steps) intervals. An eigenvalue problem of size 2,000 has then been carried out (by considering that the system has reflection symmetry) for the overall 3D flow identifying 6 eigenfunctions that carry $98 \%$ of the overall energy. In a similar fashion to what observed in the previous subsection, the first and second eigenmodes are responsible for the mass transfer between subchannels. Once again, they represent travelling waves of the type described by Eq. (11). Figure 8a shows the cross velocity for the most energetic mode while Fig. 8b shows the streamwise velocity for the same 
(a)
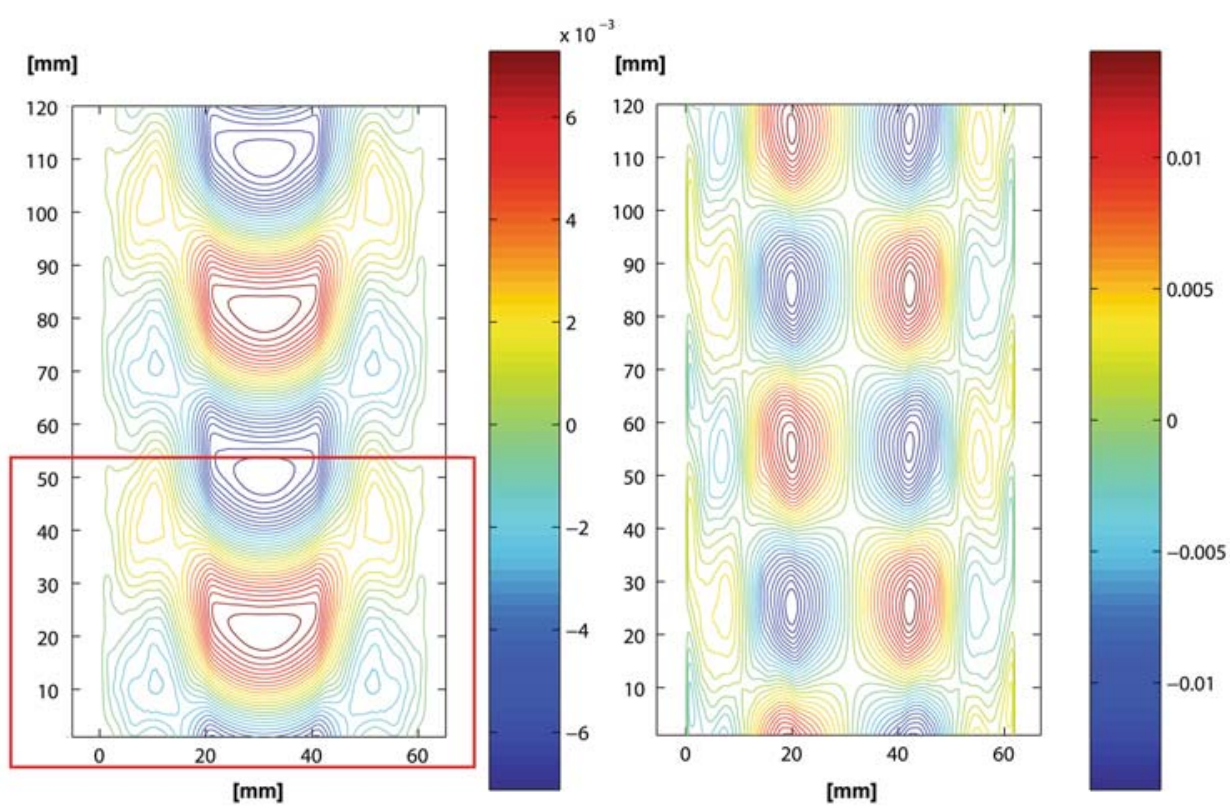

(b)

(c)
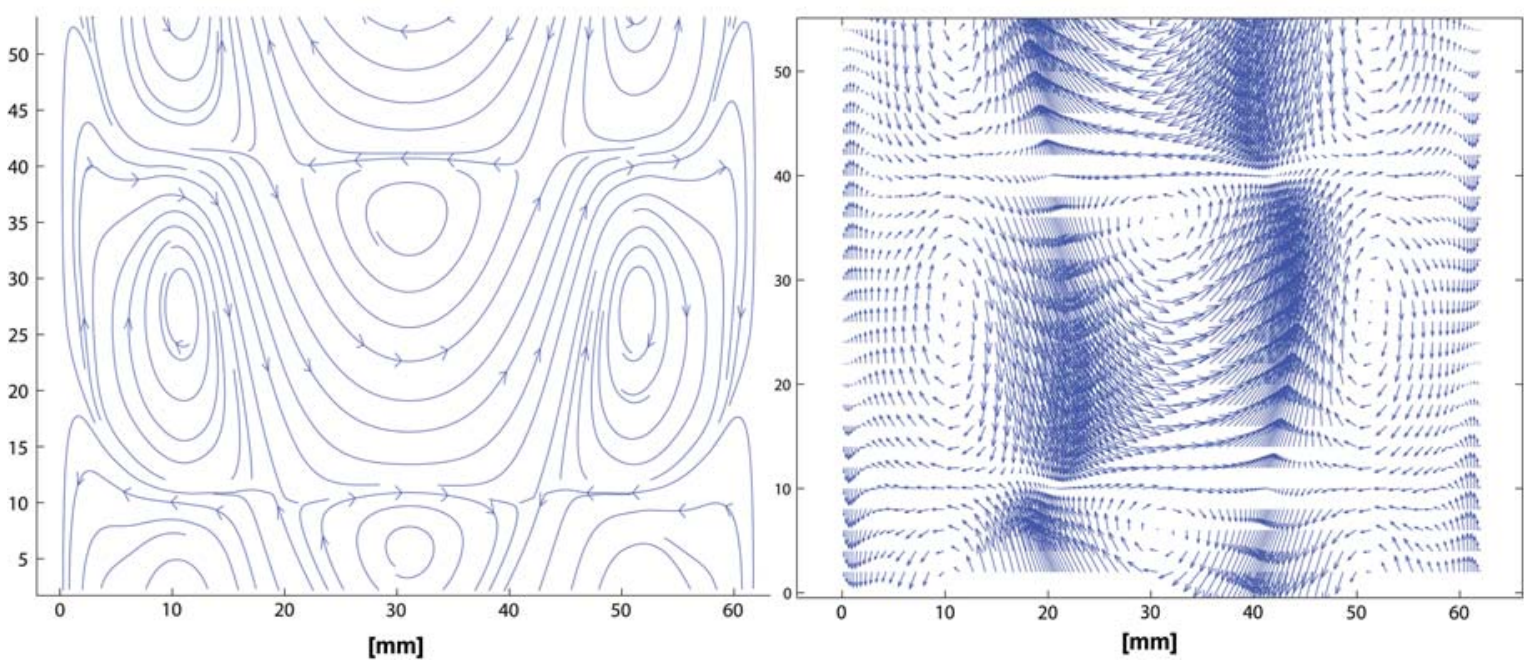

Fig. 8 Principal mode, POD of the LES computation, $R e=2,700$. a Cross velocity, b streamwise velocity, $\mathbf{c}$ detail, stream plot, d detail, vector plot

mode. Figure $8 \mathrm{c}, \mathrm{d}$ shows the streamlines and a vector plot for the most energetic mode. The similarity with the results obtained by the POD carried over experimental data is evident by comparing Figs. 5, 6, 7, and 8.

In the mode with $m=3$ (the streamlines are shown in Fig. 9), it is possible to recognize that two vortices are located near the border of the gap (similar to the mode given in Fig. 7). Modes corresponding to Fig. 6 are not present in the POD carried out over CFD data. A possible explanation is that these modes could represent a projection of the 3D travelling wave given by Fig. 8, over the measurement plane (i.e., that these are spurious modes generated by the fact that the POD is $2 \mathrm{D}$ and not fully $3 \mathrm{D}$ ).

The oscillating behaviour of the first two eigenfunctions suggests that decomposition in Fourier space rather than a direct decomposition may be more revealing. It has to be noted that a POD decomposition will always be optimal by definition even in a case with streamwise boundary conditions [16] because of the inherent properties of POD discussed on Sect. 1.1 and the space-time information it carries [17]. However, it is useful to examine which wavenumbers are involved in the oscillatory phenomenon [3]. The Fast Fourier Transform (FFT) of the velocity field has been computed and a problem of the type (1) has been solved for the Fourier coefficients. Instead of the decomposition (2), the following decomposition has been used: 


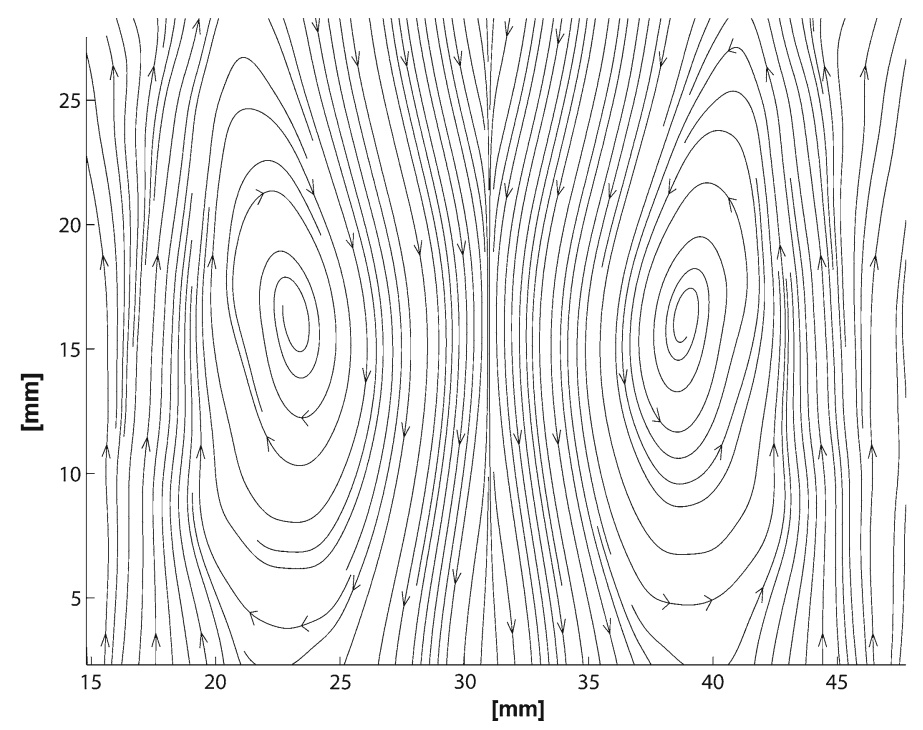

Fig. 9 Mode $m=3$, POD of the LES computation of the U-shaped channel $R e=2,700$

$$
\mathbf{u}^{\prime}(\mathbf{x})=\sum_{k} \sum_{i} a_{i} s_{i, k}(y, z) \mathrm{e}^{i k \frac{2 \pi}{L} x}
$$

where $k$ is the wavenumber, $x$ is the streamwise direction, $s_{i, k}(y, z)$ is the POD eigenfunction, $L$ is the length of the domain in the streamwise direction.

The resulting eigenvalue-eigenfunction problem has been studied for each value of the wavenumber $k$. The results are reported in Fig. 10a. The first eigenmode for $k=2$, carries more than $93 \%$ of the entire energy, while the second eigenmode carries less than $2 \%$. Fourier coefficients at $k=3$ and $k=2$ show less energetic modes. Higher wave numbers show a decreasing energy level.

The modes responsible for the mass transfer between channels are $(k=1, m=1),(k=2, m=1)$, $(k=3, m=1)$, since the value of the cross velocity is significantly grater than 0 in the narrow gap. Figure $10 \mathrm{~b}$ shows the cross velocity distribution and the streamwise velocity distribution for the mode $(k=2, m=1)$ [3]. Interestingly, this shows that in the computation more than one wavenumber is involved in the energy transfer between channels, even though only one is clearly dominant (the wavenumber $k=2$ ). Additional results concerning the POD of computational data for this geometry are available in Merzari et al. [3]. In particular, it is remarkable that the most energetic mode of turbulence $(k=2, m=1)$ is very similar in shape to the most unstable eigenmode found by the linear stability analysis of Merzari et al. [4].

\section{Eccentric channel}

An LES has been carried out for the flow in an eccentric annular channel. The approach and the code are different with the previous chapter. An in-house code has been used, where the Navier-Stokes Eqs. (5) and (6) are solved in boundary-fitted coordinates [14,18]. The Sub-Grid-Scale (SGS) stresses (7) are modelled through a dynamic model [19] where the $C_{S}$ in (8) is determined locally. Streamwise boundary conditions have been implemented. The convective fluxes have been discretized through a second-order central scheme suitable for boundary-fitted coordinates while time advancement has been carried out through an Adams-Bashforth scheme. Details of the numerical practices and details of the results are available in Merzari and Ninokata [14].

In the present work two cases have been considered. The geometry under study presents an eccentricity of 0.5 and an inner to outer diameter ratio $g=0.5$. Two Reynolds numbers $(3,200$ and 12,100$)$ have been surveyed. The grids used are $256 \times 64 \times 128$ and $256 \times 128 \times 256$, respectively (spanwise—wall-normal—streamwise).

Extensive results on the calculations carried out are reported in Merzari and Ninokata [14]. Figure 11 however shows a brief comparison for the case with $R e=3,200$ and available DNS data for channel flow [20].

In fact, the streamwise velocity near the outer wall presents a distribution comparable to a DNS of channel flow [20], confirming a previous result of Nikitin [21]. The same can be said for the turbulent kinetic energy 


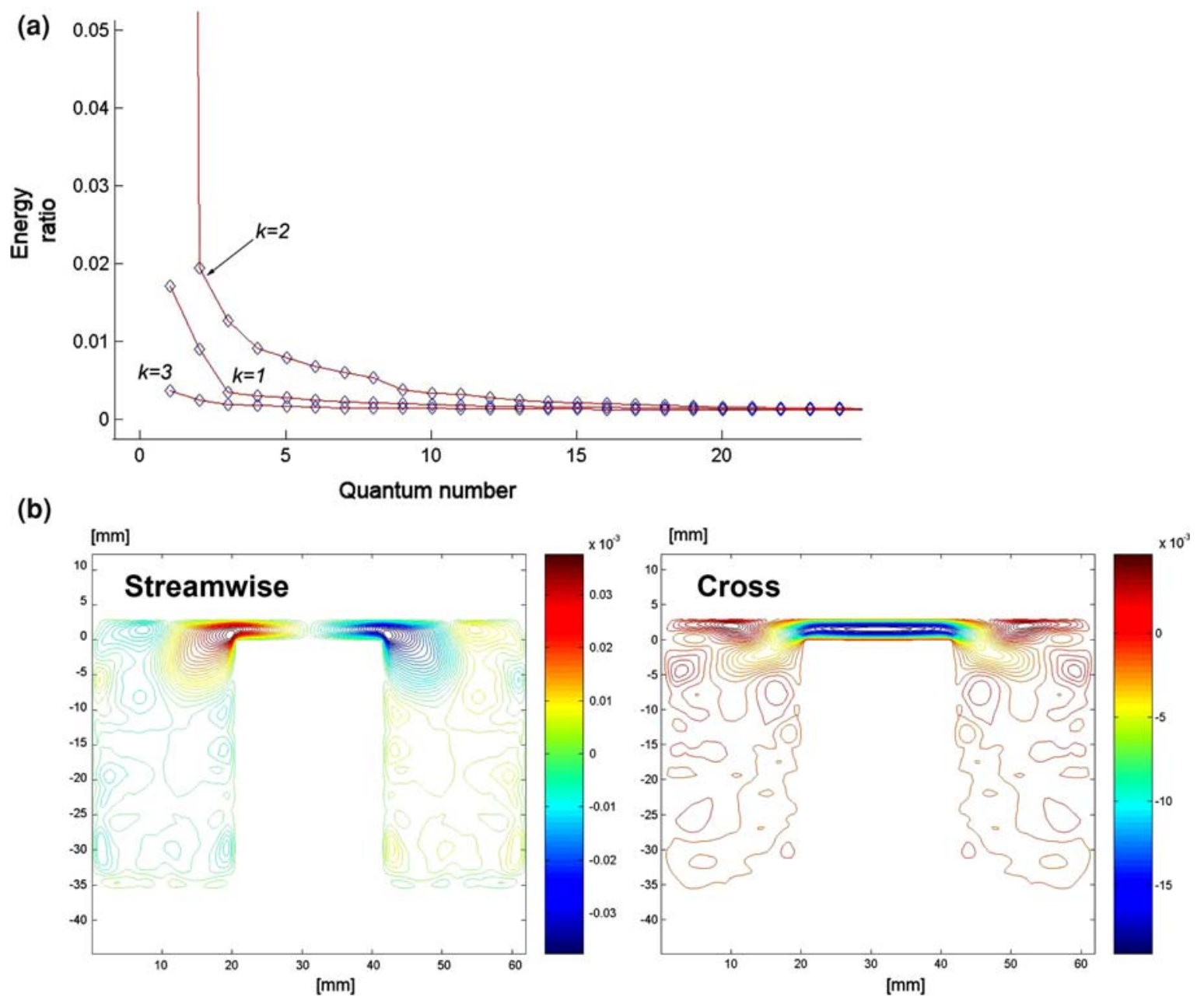

Fig. 10 a Energy content of the principal modes of the POD decomposition (from [3], computational data for the U-shaped channel at $R e=2,700)$; b Most energetic mode of turbulence $(k=2, m=1)$ (from [3])

(a)

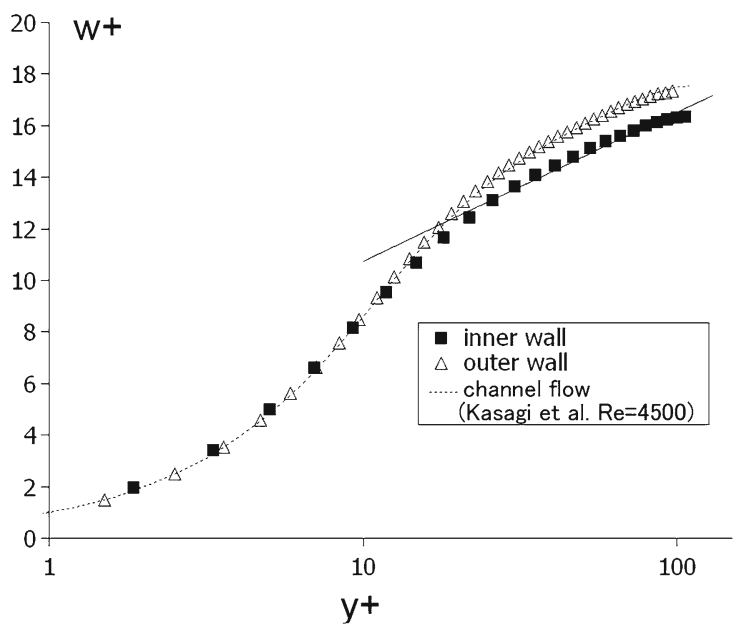

(b)

$$
\mathrm{rms}(\mathrm{w}+)
$$

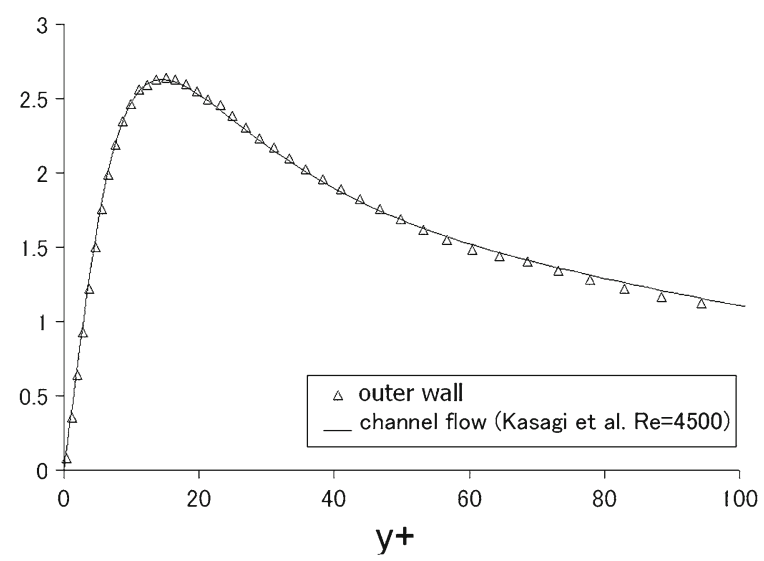

Fig. 11 Validation for the eccentric channel, $R e=3,200$, wide gap, computational data. a Streamwise velocity and $\mathbf{b}$ rms of the streamwise velocity 

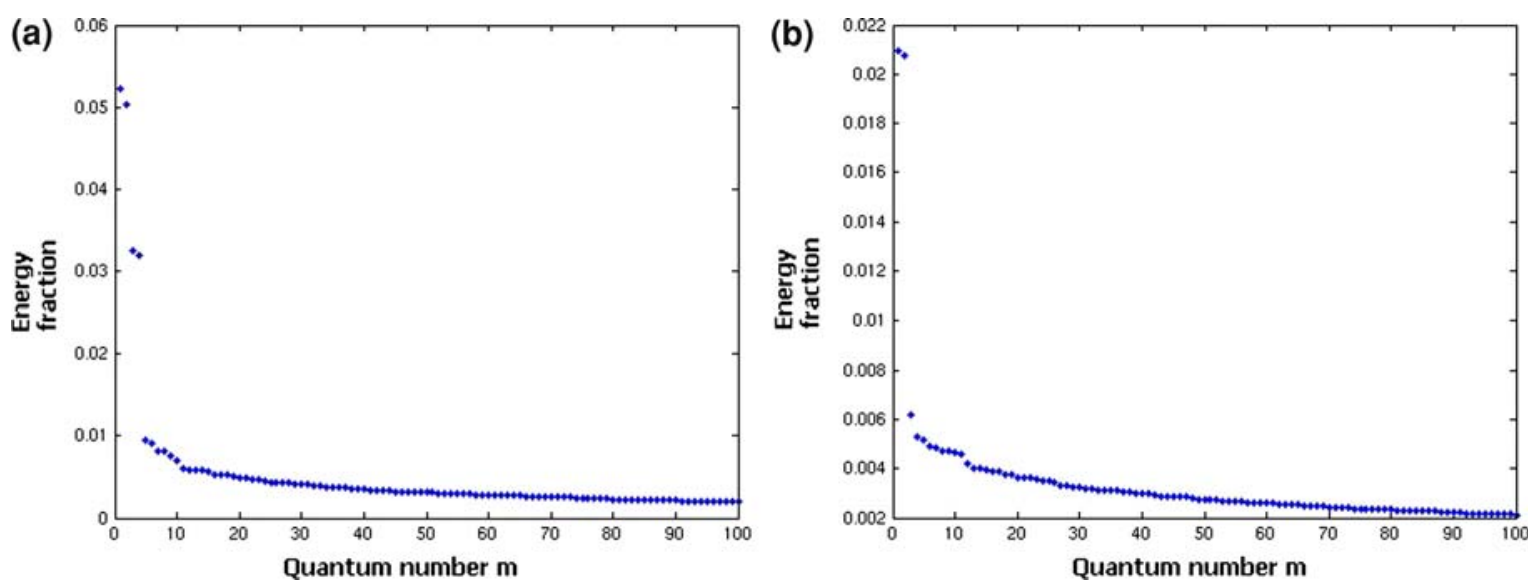

Fig. 12 Eccentric channel: energy distribution of the principal modes of the POD decomposition, computational data for the eccentric annulus. a $R e=3,200$ and $\mathbf{b} R e=12,100$
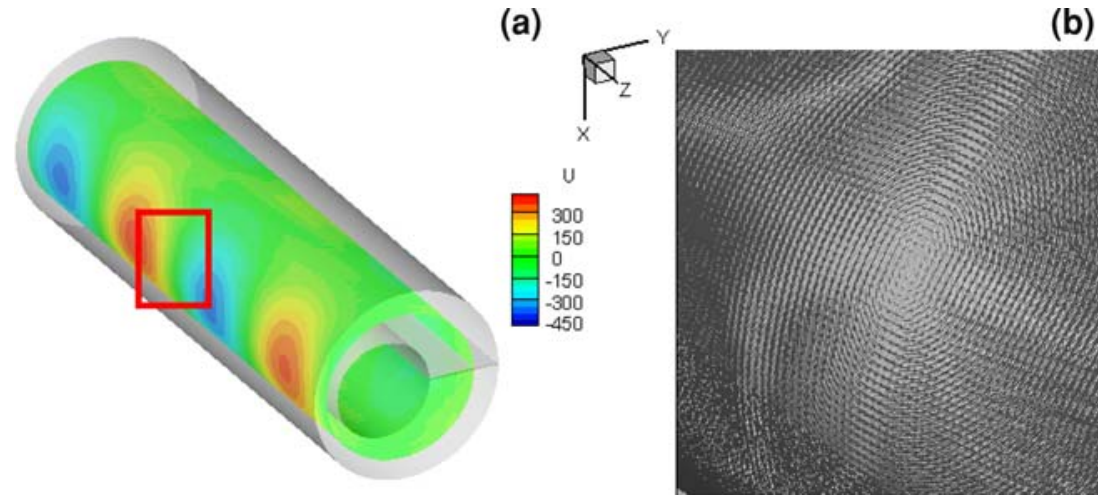

Fig. 13 Most energetic mode, normalization arbitrary, $R e=3,200$, computational data. a Cross velocity, $\mathbf{b}$ vector plot—detail

and the rms of the streamwise velocity component. Secondary flows are observed in the cross-section and they are similar in shape to the ones proposed by Nikitin [21]: the flow is transported from the region of higher momentum (wide gap) to the narrow gap along the walls and it is transported back to the wide gap in the region of low shear (mid-distance from the cylinders) [14].

\subsection{POD}

The POD has been carried out at two Reynolds numbers $(R e=3,200$ with a domain length of $4 \pi$ and $R e=12,100$ with a domain length equal to $2 \pi$ ) with 2,000 snapshots each. In both cases the snapshots were sampled at $0.015 \mathrm{~s}$ intervals. Figure 12a shows that, for the case Reynolds number equal to 3,200, the first 6 modes contain $20 \%$ of the total energy while Fig. 12b shows that at higher Reynolds number the 6 most energetic modes contain approximately 6\% of the total energy. This indicates that an energy transfer occurs between the most energetic modes and the other modes (Fig. 12) as the Reynolds number increases.

The first four modes in the case with $R e=3,200$ and the first two modes in the case at $R e=12,100$ are representative of the same type of travelling wave encountered in the previous section. For the case at $R e=3,200$ two wavelengths appear to be present as it appears evident from Figs. 13 and 14. In fact the use of periodic boundary conditions and a finite computational length in the streamwise direction imply a discretized wavenumber spectrum and subsequently the impossibility for the computation to reproduce exactly the correct wavenumber spectrum (unless the domain is extremely long or it is chosen within a certain range, in the sense discussed in [3]). These issues are discussed extensively in Merzari et al. [3]. 
(a)

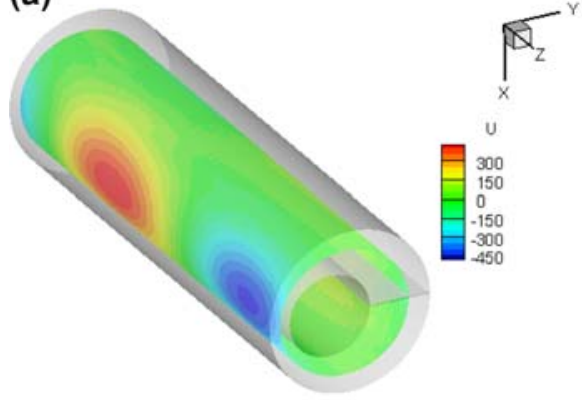

(b)

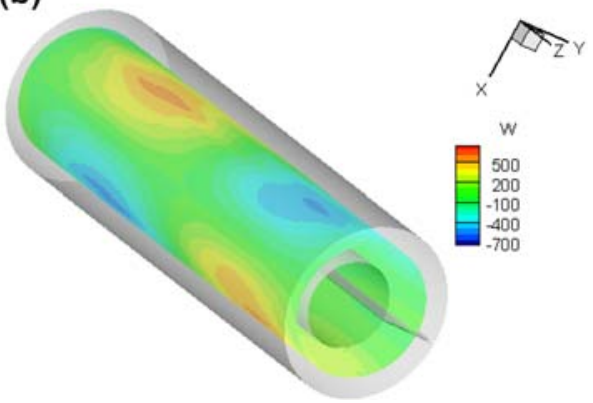

Fig. 14 Mode with quantum number $m=3$, normalization arbitrary, $R e=3,200$, computational data. a Cross velocity, b Streamwise velocity
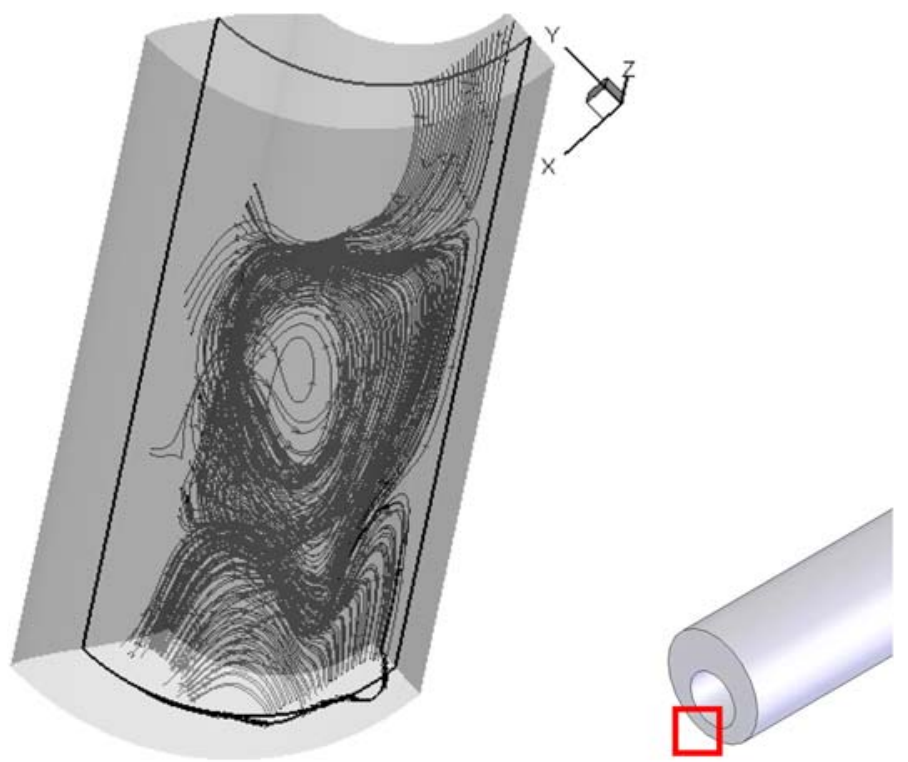

Fig. 15 Streamlines for the mode with quantum number, $m=6, R e=3,200$, computational data

For $R e=3,200$, the modes corresponding to quantum numbers $m=4$ and $m=5$ contain a series of vortices on the edges of the gap (Fig. 15), similar to the modes shown in Fig. 7. Similar modes have been found to be dominant at higher Reynolds number.

In conclusion, the POD conducted on eccentric channels shows that travelling wave modes are present in this geometry as well, but they are considerably less energetic than the case considered in the previous section. Moreover, as the Reynolds number increases they appear to be less dominant toward other modes. From what shown in other works [14] this trend might be different for different values of the geometric parameters. In particular for higher eccentricity, the oscillations have been found to be dominant at higher Reynolds numbers (e.g., for $e=0.8$ they are present at $R e=11,000$ ).

\section{Dynamics}

In this section, the underlying dynamics of the oscillations will be examined. POD can be used to decompose the flow field into a series of modes as shown by Eq. (2). The Navier-Stokes equations can then be rewritten in a set of ODEs of the type:

$$
\frac{\mathrm{d} a_{i}}{\mathrm{~d} t}=\sum_{j k} A_{i j k} a_{j} a_{k}+\sum_{i} B_{i j} a_{j}+C_{i}
$$


Each obtained by projecting an orthogonal eigenfunction $\boldsymbol{\sigma}_{\mathbf{i}}$ onto the momentum equation. In the momentum equation the fluctuation $\mathbf{u}^{\prime}(\mathbf{x})$ of the velocity toward the average can be truncated as:

$$
\mathbf{u}^{\prime}(\mathbf{x})=\sum_{i=1, N_{e q}} a_{i} \sigma_{\mathbf{i}}(\mathbf{x})
$$

thus reducing (13) into a set of $N_{\text {eq }}$ ODEs. It is worth noticing that often an additional cubic term [8,22] is added to (13). The coefficients $A_{i j k}, B_{i j}$ and $C_{i}$ in (13) are determined by the spatial distribution of the eigenfunctions and they are computed numerically using third-order quadrature schemes. The complete expressions for the coefficients are available in Appendix A.

The problem can also be addressed in an alternative way by considering the decomposition in Fourier space (12) instead of (2). The expression for the coefficients and the POD eigenfunctions change, and so does the dynamics, that is more complex [23]. In this work, however, only the POD in physical space with the coefficients given by Appendix A will be considered, because of its simplicity and more immediate physical meaning.

Another important issue is the introduction of a necessary closure to take into account all the scales of turbulence (since the series (14) does not contain all the turbulent kinetic energy if $N_{\text {eq }}$ is finite). This has been widely discussed by Cazemier [24]. An eddy viscosity approach has been employed here.

\subsection{Coefficient analysis}

At first, let us examine the general properties of the system (13) generated by a decomposition of the type (2) which has the advantage of not including the average field (i.e., letting us examine the influence of the Reynolds number separately).

The primary interest of this analysis is in obtaining some insight into the physics of the oscillations starting from the value of the computed coefficients. The properties discussed in the following have been verified for all the PODs performed in this work, but, in the interest of brevity, they will be detailed only for the system of ODEs obtained from the POD carried out over experimental data.

One interesting property of the system is that it can be rewritten into two systems non-linearly coupled (by reordering the equations):

$$
\begin{gathered}
\frac{\mathrm{d} a_{i}}{\mathrm{~d} t}=\sum_{j k} A_{i j k} a_{j} a_{k}+\sum_{j=1}^{M_{\mathrm{eq}}} B_{i j} a_{j}, \quad \text { for } j<M_{\mathrm{eq}}+1 \\
\frac{\mathrm{d} a_{i}}{\mathrm{~d} t}=\sum_{j k} A_{i j k} a_{j} a_{k}+\sum_{j=M_{\mathrm{eq}}+1}^{N_{\mathrm{eq}}} B_{i j} a_{j}+C_{i}, \quad \text { for } j>M_{\mathrm{eq}}, \quad j<N_{\mathrm{eq}}+1
\end{gathered}
$$

because of the properties of the computed matrix $B_{i j}$ and the computed vector $C_{i}$. The first equation represents the dynamics of modes of the type shown in Figs. 5 and 6 (i.e., the modes responsible for the mass flow exchange between subchannels). The second equation represents the dynamics of the modes of the type shown in Fig. 7.

Another interesting property is that the non-linear terms are up to 20 times smaller than the linear terms. If the non-linear terms are neglected the system given by Eq. (13) can therefore be separated into two uncoupled linear systems:

$$
\begin{gathered}
\frac{\mathrm{d} a_{i}}{\mathrm{~d} t}=\sum_{j=1}^{M_{\mathrm{eq}}} \bar{B}_{i j} a_{j}, \quad \text { for } j<M_{\mathrm{eq}}+1 \\
\frac{\mathrm{d} a_{i}}{\mathrm{~d} t}=\sum_{j=M_{\mathrm{eq}}+1}^{N_{\mathrm{eq}}} \overline{\bar{B}}_{i j} a_{j}+C_{i}, \quad \text { for } j>M_{\mathrm{eq}}, \quad j<N_{\mathrm{eq}}+1
\end{gathered}
$$


Table 1 Eigenvalue spectrum for the linear matrices in Eq. (16.1) and (16.2)

\begin{tabular}{ll}
\hline Eigenvalue spectrum of $\bar{B}_{i j}$ & Eigenvalue spectrum of $\overline{\bar{B}}_{i j}$ \\
\hline $0+6.5304 i$ & $0.0+1.4178 i$ \\
$0-6.5304 i$ & $0.0-1.4178 i$ \\
$-0.1279+1.3626 i$ & $0.0+0.5658 i$ \\
$-0.1279-1.3626 i$ & $0.0-0.5658 i$ \\
$-0.0841+0.6703 i$ & $-0.0098+0.0439 i$ \\
$-0.0841-0.6703 i$ & $-0.0098-0.0439 i$ \\
$-0.0497+0.0233 i$ & $-0.0141+0.2845 i$ \\
$-0.0497-0.0233 i$ & $-0.0141-0.2845 i$ \\
\hline
\end{tabular}

The coefficients are obtained from the POD carried out over experimental data at $R e=2,000$

The eigenvalues of the two linear matrices $\overline{B_{i j}}$ and $\overline{\overline{B_{i j}}}$ are given in Table 1 considering the first 16 eigenmodes (91\% of the Turbulent Kinetic Energy) for the experimental POD. The equations given by (16.1) have the trivial solution:

$$
a_{i}(t)=\sum_{j=1}^{M_{\mathrm{eq}}} b_{i j} \exp \left(\lambda_{j} t\right)
$$

where $\lambda_{j}$ are the eigenvalues of the matrix $\bar{B}_{i j}$. Thus, it is interesting to notice that only two eigenvalues have an almost zero real component, and all real components of the eigenvalues are negative. A stable oscillatory mode thus exists whose frequency depends upon the value of the Reynolds number. Let us examine in detail matrix $B_{i j}$ (Appendix A). It can, in all generality, be written as:

$$
B_{i j}=B_{i j}^{1}+v B_{i j}^{2}
$$

where $B_{i j}^{1}$ depends upon the value of the mean velocity and thus depends on the Reynolds numbers (whereas $B_{i j}^{2}$ is independent of the Reynolds number). $B_{i j}^{1}$ has several pure imaginary eigenvalues while $B_{i j}^{2}$ has only real eigenvalues. Moreover, for $R e=2,000$, the value of the imaginary part of the strongest eigenvalue is of the same order of $2 \pi f$, where $f$ is the experimental frequency of the oscillations and does not depend on the size of $B_{i j}$ (i.e., it does not depend on $N_{\text {eq }}$ if $N_{\text {eq }}>8$ ).

The structure of the eigenvalues of $B_{i j}$ accounts for the presence of a threshold. In fact at low Reynolds numbers $B_{i j}^{1}$ is small (since the bulk velocity is small) and as a consequence the eigenvalues of $B_{i j}$ will be essentially similar to $B_{i j}^{2}$. This corresponds to the fact that, at low Reynolds number, the viscous dumping does not allow for the propagation of the wave and the oscillations die out quickly.

At higher Reynolds number the eigenvalues of $B_{i j}$ coincide with $B_{i j}^{1}$, moreover, the imaginary part of the strongest eigenvalue grows linearly with the Reynolds number (if we assume the velocity in the region to grow linearly) thus explaining the dependence of frequency upon the Reynolds number.

Since $B_{i j}^{1}$ represents the coupling between each mode and the mean flow it is possible to conclude that the oscillations are transported by linear interaction between modes of the type presented in Fig. 5 and the mean flow. The modes represented in Fig. 5 are thus propagated in a travelling wave-like manner by the mean streamwise flow.

\subsection{Solution of POD equations}

Since the two systems are uncoupled linearly, the non-linear coupling is essential to represent the whole physics. The Eq. (13) have been solved by numerical integration using higher order Runge-Kutta schemes [25]. The results give qualitative agreement with experimental data. In particular, a travelling wave is clearly present, propagating in the streamwise direction with constant speed.

Similar results have been obtained for all cases considered in this work (eccentric channel, rectangular channels connected by a narrow gap - both computational and experimental data). In spite of the severe simplifications made in Sect. 2, even the modes obtained through the experimental POD are able to describe qualitatively well the dynamics of the system. 
(a)

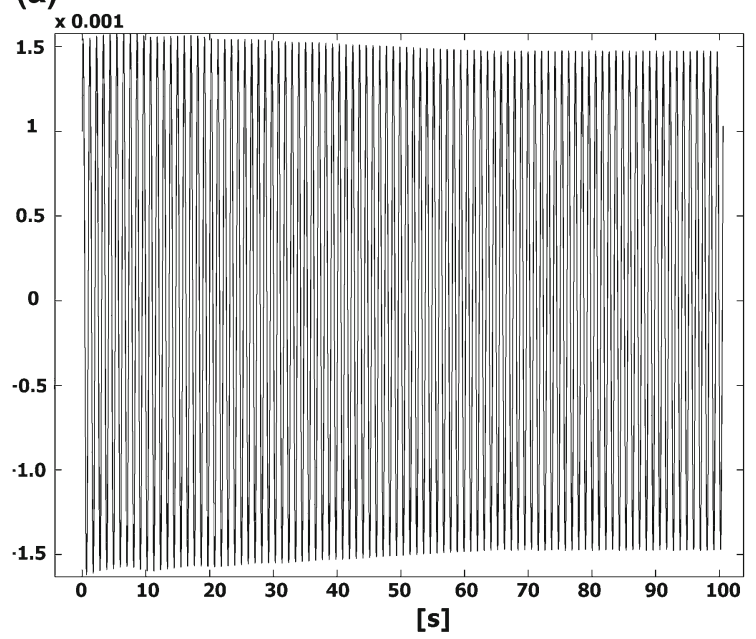

(c)

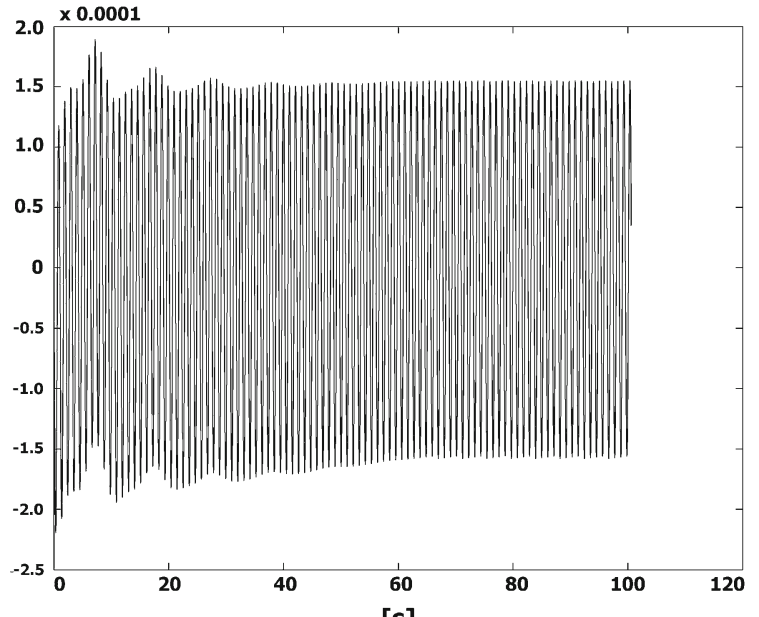

(e)

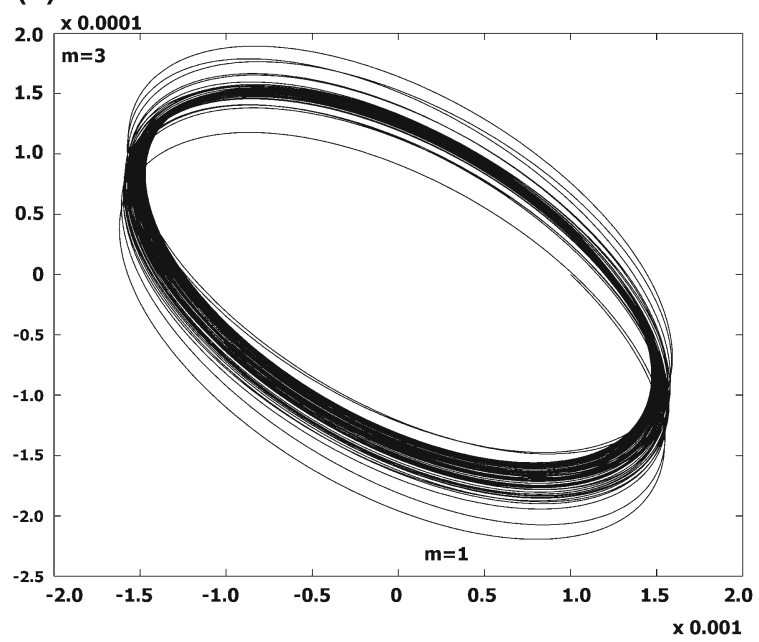

(b)

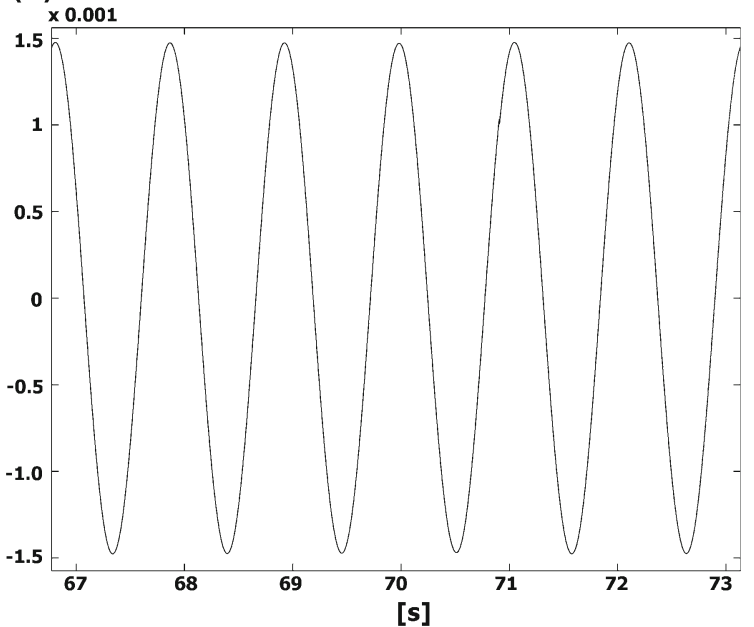

(d)

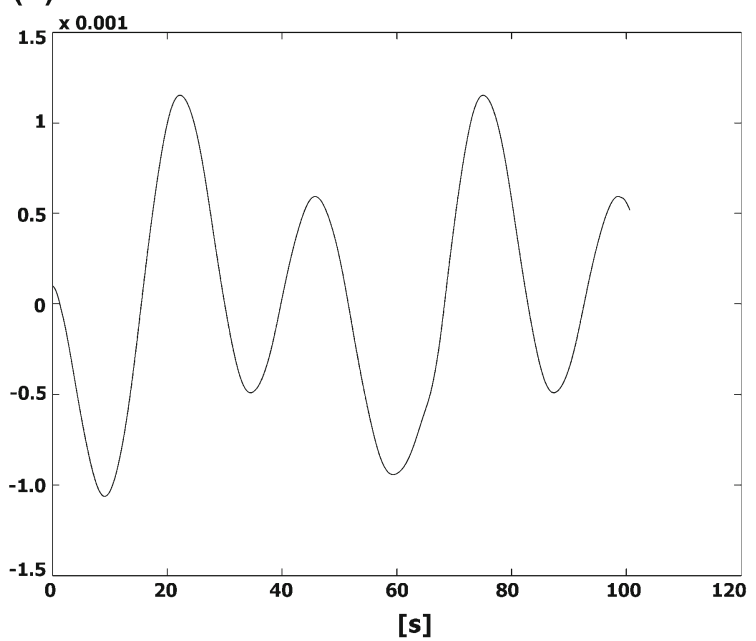

(f)

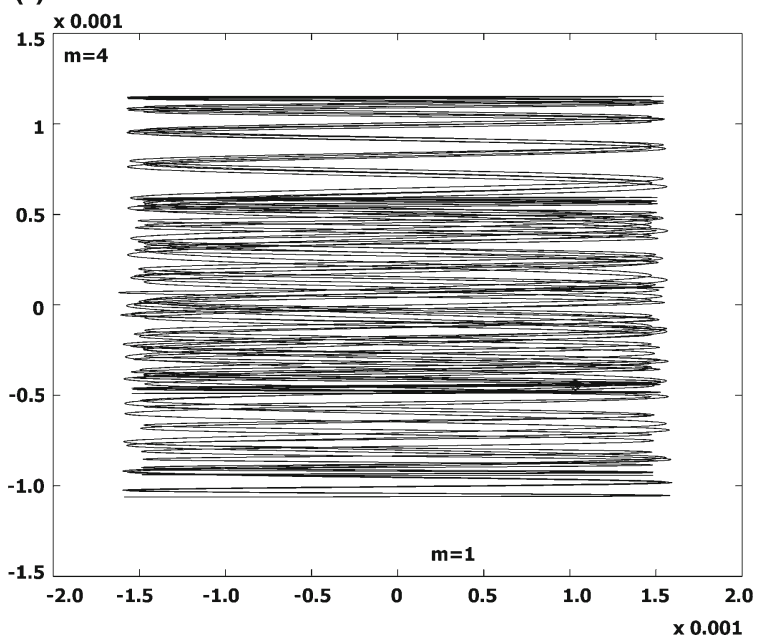

Fig. 16 Solution of the ODE set obtained from the POD of experimental data. a Temporal evolution of the coefficient of the most energetic mode. $\mathbf{b}$ Detail of the temporal evolution of the most energetic mode. $\mathbf{c}$ Temporal evolution of the mode with $m=3$. d Temporal evolution of the mode with $m=4$. e Phase dynamic between the mode with $m=1$ and $m=3$. f Phase dynamic between the mode with $m=1$ and $m=4$ 
(a)

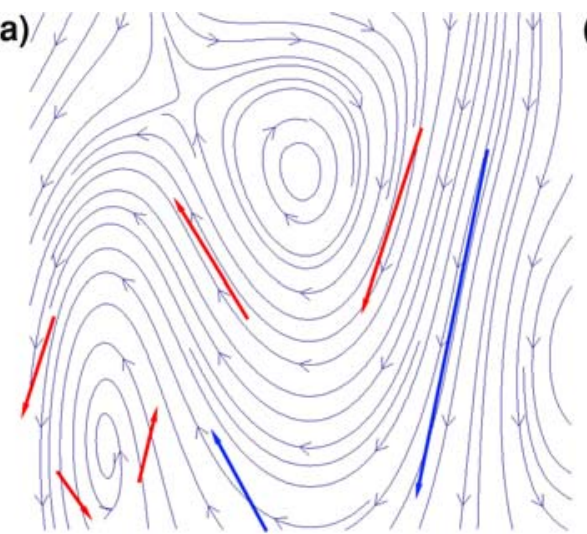

(b)

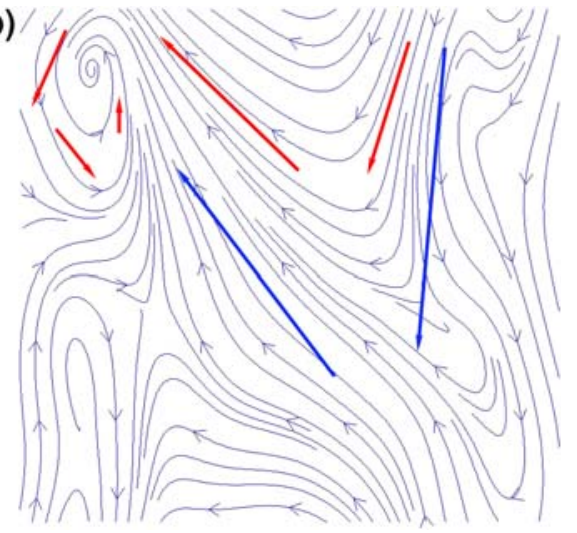

Fig. 17 Streamline plots. Low-dimensional model (a), experiment (b)

As an example, we report in the following the results for a dynamical system obtained by the POD carried out over experimental data $\left(N_{\mathrm{eq}}=16\right)$.

Figure 16 shows phase plots and time histories for some of the modes. Figure 16a represents the time history for the most energetic mode $(m=1)$, while Fig. 16b shows a detail of the same time history. Figure 16c represent the time history of the mode with $m=3$ and Fig. 16d of the mode with $m=4$. Figure 16d, e shows the phase coupling between different types of modes:

1. Figure $16 \mathrm{~d}$ shows the phase interaction between two oscillatory modes (system 15.1 with non-linear coupling);

2. Figure $16 \mathrm{~b}$ shows the interaction between an oscillatory mode $(m=1)$ and a mode with $m=4$.

Figure 17 shows the comparison between a stream plot obtained by solving the non-linear system (13) and an experimental stream plot for two rectangular channels connected by a narrow gap. The stream plot for the POD appears to be simpler but at the same time, qualitative features of the flow are reproduced (the arrows represent typical flow paths in the experiment and the POD).

\section{Conclusions}

Through the statistical analysis of numerical and experimental data, the present work proposes a POD-based approach for the studying of the long-standing problem of flow pulsations between channels connected by a narrow gap.

Experimental and computational data have been used in different geometry and for different Reynolds numbers. The flow has been decomposed, in the physical space, and then in Fourier space, into a series of eigenmodes. In all the cases examined the eigenmodes structure is similar, indicating that the properties discussed here are inherent to the flow in geometry containing a narrow gap.

The modes associated with the flow oscillations have been identified. It has been confirmed by the experimental observation that the flow is dominated by a single wavenumber in Fourier space. In fact, the POD carried out on experimental and numerical data shows that a travelling wave is present in the region of the narrow gap in all cases examined. Such travelling waves are very similar in shape to the most unstable eigenmode found by the linear stability analysis of Merzari et al. [4]. An additional mode containing alternating vortices on the edge of the gap is also present (Fig. 7).

The dynamics of the oscillations seems to be driven by the coupling between the average flow field (and notably the secondary flows) and the modes representing the travelling waves (Figs. 5, 8, 13). Moreover, the dependency of the frequency oscillations as well as the Reynolds threshold effect on the Reynolds number can be predicted by eigenvalue decomposition of the linear coefficient matrix $B_{i j}$ (Sect. 4).

In the case of eccentric channels, two travelling waves of different wavelength were present. This is a numeric effect, due to the use of periodic boundary conditions in the streamwise direction (which implies a discretized wavenumber space [3]). Moreover, for the geometric parameters studied here $(g=0.5, e=0.5)$, as the Reynolds increases the oscillatory modes appear to be less dominant toward other modes. 
Independently from this work, the statistical decomposition of the flow through a POD technique carries out some significance: it can in fact be used to analyze the consistency of a numerical simulation with experimental observations [3]. The dominant wavenumber present in the numerical simulation should be sufficiently close to the experimental wavenumber to correctly reproduce the dynamic of the system.

Future work should entail the extension of the present methodology to more complex geometry (e.g., rod bundles) where the pattern of the oscillations is inherently 3D. Another important area of investigation would be the laminar-turbulent transition in eccentric channels or other simplified geometries. In particular it would be interesting to examine in further detail how the bifurcation found by Gosset and Tavoularis [5] and Merzari et al. [4] develops into the travelling waves shown here. An in-depth analysis of the mechanics of the energy transfer between coherent modes and incoherent modes should also be carried out in order to clarity the effect of the geometric parameters.

\section{Appendix A}

Coefficients of Eq. (14):

$$
\begin{aligned}
A_{i j k} & =-\left(\boldsymbol{\sigma}_{i}, \boldsymbol{\sigma}_{j} \cdot \nabla \boldsymbol{\sigma}_{k}\right) \\
B_{i j} & =-\left(\boldsymbol{\sigma}_{i},\langle\mathbf{u}\rangle \cdot \nabla \boldsymbol{\sigma}_{j}\right)-\left(\boldsymbol{\sigma}_{i}, \boldsymbol{\sigma}_{j} \cdot \nabla\langle\mathbf{u}\rangle\right)+v\left(\boldsymbol{\sigma}_{\boldsymbol{i}}, \Delta \boldsymbol{\sigma}_{j}\right) \\
C_{i} & =-\left(\boldsymbol{\sigma}_{\boldsymbol{i}},\langle\mathbf{u}\rangle \cdot \nabla\langle\mathbf{u}\rangle\right)+v\left(\boldsymbol{\sigma}_{\boldsymbol{i}}, \Delta\langle\mathbf{u}\rangle\right)-\left(\boldsymbol{\sigma}_{\boldsymbol{i}}, \nabla\left(\langle p\rangle+p^{\prime}\right)\right)
\end{aligned}
$$

$B_{i j}$ can be rewritten as in Eq. (18), where:

$$
\begin{aligned}
& B_{i j}^{1}=-\left(\sigma_{i},\langle\mathbf{u}\rangle \cdot \nabla \sigma_{j}\right)-\left(\sigma_{i}, \sigma_{j} \cdot \nabla\langle\mathbf{u}\rangle\right) \\
& B_{i j}^{2}=\left(\sigma_{i}, \Delta \sigma_{j}\right)
\end{aligned}
$$

where clearly only the first term depends on the averaged streamwise velocity profile. The pressure term present in (A3) can be rewritten considering that the POD expansion is a linear combination of flow realizations (and therefore solution of the continuity equation):

$$
\left(\sigma_{i}, \nabla\left(\langle p\rangle+p^{\prime}\right)\right)=\left(\sigma_{i}, \nabla(\langle p\rangle)\right)+\left(\operatorname{div}\left(\sigma_{i}\right), p^{\prime}\right)+(\text { b.t. })=\left(\sigma_{i}, \nabla(\langle p\rangle)\right)+(\text { b.t. })
$$

where (b.t.) is a boundary term and depends on the computational domain and the boundary conditions. In case of Dirichlet boundary conditions or fully developed (cyclic) boundary conditions it is rigorously equal to zero, and generally speaking it is negligible if compared to other terms $[8,22]$. An extensive derivation can be found in Cazemier [24].

\section{References}

1. Hooper, J.D., Rehme, K.: Large-scale structural effect in developed turbulent flows through closely-spaced rod arrays. J. Fluid Mech. 145, 305-337 (1984)

2. Meyer, L., Rehme, K.: Large-scale turbulence phenomena in compound rectangular channels. Exp. Therm. Fluid Sci. 8, 286-304 (1994)

3. Merzari, E., Ninokata, H., Baglietto, E.: numerical simulation of the flow in tight-lattice fuel bundles. Nucl. Eng. Des. 238, 1703 (2008)

4. Merzari, E., Wang, S., Ninokata, H., Theofilis, V.: Biglobal linear stability analysis for the flow in eccentric annular channels and a related geometry. Phys. Fluids 20, 114104 (2008) doi:10.1063/1.3005864

5. Gosset, A., Tavoularis, S.: Laminar flow instability in a rectangular channel with a cylindrical core. Phys. Fluids 18, 044108 (2006)

6. Guellouz, M.S., Tavoularis, S.: The structure of turbulent flow in a rectangular channel containing a cylindrical rod—Part I: Reynolds averaged experiments. Exp. Therm. Fluid Sci. 23, 59 (2000)

7. Lexmond, A., Mudde, M., Van der Haagen M.: Visualization of the vortex street and characterization of the cross flow in the gap between two subchannels. In: Proceedings of 11th Nureth. Avignone, Paper 122 (2005)

8. Aubry, N., Holmes, P., Lumley, J.L., Stone, E.: The dynamics of coherent structures in the wall region of a turbulent boundary layer. J. Fluid Mech. 192, 115-173 (1988)

9. Sanghi, S., Aubry, N.: Low dimensional models for the structure and dynamics in near wall turbulence. J. Fluid Mech. 247, 455-488 (1993)

10. Lumley, J.L.: Stochastic Tools in Turbulence. Academic Press, New York (1970)

11. Berkooz, G., Holmes, P., Lumley, J.L.: The proper orthogonal decomposition in the analisys of turbulent flow. Ann. Rev. Fluid Mech. 25, 539-575 (1993) 
12. Sirovich, L.: Turbulence and the dynamics of coherent structures, Part I: Coherent structures. Q. Appl. Math. XLV, 561$571(1987)$

13. Aubry, N., Guyonnet, R., Lima, R.: Spatiotemporal analysis of complex signals: theory and applications. J. Stat. Phys. 64, 683-739 (1991)

14. Merzari, E., Ninokata, H.: Anisotropic turbulence and coherent structures in eccentric annular channels. Flow Turbul. Combust. 82, 93-120 (2009). doi:10.1007/s10494-008-9170-2

15. Deardorff, J.W.: A numerical study of three-dimensional turbulent channel flow at large Reynolds numbers. J. Fluid Mech. 41, 453-480 (1970)

16. Aubry, N.: On the hidden beauty of the proper orthogonal decomposition. Theor. Comput. Fluid Dyn. 2, 339-352 (1991)

17. Aubry, N., Guyonnet, R., Lima, R.: Spatio-temporal symmetries and bifurcations via bi-orthogonal decompositions. J. Nonlinear Sci. 2, 183-215 (1992)

18. Zang, Y., Street, R.L., Koseff, J.R.: A non-staggered grid, fractional step method for time-dependent incompressible Navier-Stokes equations in curvilinear coordinates. J. Comput. Phys. 114, 1 (1994)

19. Jordan, S.A.: Dynamic subgrid-scale modeling for large-eddy simulations in complex topologies. J. Fluids Eng. 123(3), 619-627 (2001)

20. Kasagi, N., Horiuti, K., Miyake, Y., Miyauchi, T., Nagano, Y.: Establishment of the direct numerical simulation data bases of turbulent transport phenomena. Available on the Website of Tokyo University. http://www.thtlab.t.u-tokyo.ac.jp/

21. Nikitin, N.V.: Direct numerical simulation of turbulent flows in eccentric pipes. Comput. Math. Math. Phys. 46, 509-526 (2006)

22. Omurtag, A., Sirovich, L.: On low-dimensional modeling of channel turbulence. Theor. Comput. Fluid Dyn. 13, 115-127 (1999)

23. Merzari, E., Ninokata, H.: Toward a dynamical system approach for the understanding of turbulent flow pulsation between subchannels. In: Proceedings of the 12th Nureth. Pittsburgh, USA (2007)

24. Cazemier, W.: Proper orthogonal decomposition and low dimensional models for turbulent flow. $\mathrm{PhD}$ thesis, University of Groningen, Groningen, The Netherlands (1997). ISBN 90-367-0682-3

25. Shampine, L.F., Reichelt, M.W.: The MATLAB ODE suite. SIAM J. Sci. Comput. 18, 1-22 (1997) 